\title{
Location of Periventricular Nodular Heterotopia Is Related to the Malformation Phenotype on MRI
}

\author{
G. González, L. Vedolin, B. Barry, A. Poduri, C. Walsh, and A.J. Barkovich
}

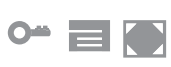

\begin{abstract}
BACKGROUND AND PURPOSE: Periventricular nodular heterotopia are common malformations of cortical development that are associated with many clinical syndromes and with many different neuroimaging phenotypes. The purpose of this study was to determine whether specific malformation phenotypes may be related to location, side, or number of PNH as assessed by MR imaging.
\end{abstract}

MATERIALS AND METHODS: MR images of 200 patients previously diagnosed with PNH were retrospectively analyzed. PNH were classified according to their location along the ventricles (anterior, posterior, or diffuse), side (unilateral or bilateral), and number of nodules $(<5,6-10$, or $>10)$. The cerebrum, brain stem and cerebellum were analyzed to assess associated anomalies. Associations between PNH location and the presence of other anomalies were tested by using Fisher exact test and $\chi^{2}$ test.

RESULTS: Posterior PNH were significantly associated with malformations of the cerebral cortex, diminished white matter volume, and mid-/hindbrain anomalies. Diffuse PNH were associated with diminished white matter volume, callosal "anomalies," and the presence of megacisterna magna. Unilateral PNH were strongly associated with cortical malformations.

CONCLUSIONS: Certain malformation complexes are associated with PNH in specific locations: posterior PNH with cerebral cortical and mid-/hindbrain malformations and diffuse PNH with callosal anomalies and megacisterna magna. Knowledge of these associations should allow more directed analyses of brain MR imaging in patients with PNH. In addition, knowledge of these associations may help to direct studies to elucidate the causes of these malformation complexes.

ABBREVIATIONS: $\mathrm{aPNH}=$ anterior periventricular nodular heterotopia; $\mathrm{CC}=$ corpus callosum; $\mathrm{dPNH}=$ diffuse periventricular nodular heterotopia; $\mathrm{PNH}=$ periventricular nodular heterotopia; $\mathrm{PPNH}=$ posterior periventricular nodular heterotopia

$\mathrm{H}_{\mathrm{i}}$ eterotopia represent malformations of cortical development in which neurons do not migrate to their proper final location. ${ }^{1}$ They are broadly classified by location as PNH, subcortical heterotopia, and leptomeningeal heterotopia, the latter currently being difficult to detect by imaging. ${ }^{2} \mathrm{PNH}$ are the most common group, being identified as variably sized nodules of neurons along the surface of lateral ventricles on imaging or postmortem studies. Histologic analysis shows these nodules to exhibit rudimentary lamination, resembling that in the cortex. ${ }^{3}$ Traditionally, $\mathrm{PNH}$

Received June 22, 2012; accepted after revision July 19.

From the Department of Radiology and Biomedical Imaging (G.G., L.V., A.J.B.), University of California, San Francisco, California; Department of Radiology (G.G.), Hospital San Juan de Dios, Santiago, Chile; Neuroradiology Section (L.V.), Hospital Moinhos de Vento, Porto Alegre, Brazil; Division of Genetics (B.B., C.W.), Manton Center for Orphan Disease Research and Howard Hughes Medical Institute, Boston Children's Hospital, Boston, Massachusetts; Department of Neurology (A.P., C.W.), Boston Children's Hospital, Boston, Massachusetts; and Department of Neurology (C.W.), Harvard Medical School, Boston, Massachusetts.

C.W. and A.J.B. were supported by grants from the NINDS (R01-NS35129); A.P. was supported by the NINDS (K23NS069784).

Paper previously presented at: 50th Annual Meeting of the ASNR and the Foundation of the ASNR Symposium; April 21-26, 2012; New York, New York. have been considered a result of impaired neuronal migration ${ }^{4}$; however, recent evidence suggests that the primary cause may be a disruption of the neuroependyma, which impairs postmitotic neurons from attaching to radial glial cells and, therefore, impairs initiation of the migration process. ${ }^{5,6}$

PNH may be found incidentally in asymptomatic patients; more frequently, they are discovered after imaging is performed for delayed development or epilepsy. ${ }^{7-10}$ Response to epilepsy treatment is variable, ranging from good control with antiepileptic drugs to intractable epilepsy. Patients with no other cortical disorders often have normal intelligence or mild intellectual disability. However, those patients with associated malformations can have developmental delay of variable severity.

Please address correspondence to Guido González, MD, Department of Radiology and Biomedical Imaging, University of California, San Francisco, 505 Parnassus Ave, San Francisco, CA, 94143; e-mail: guido.gonzalez7@gmail.com

- Indicates open access to non-subscribers at www.ajnr.org

$\equiv$ Indicates article with supplemental on-line tables.

Indicates article with supplemental on-line figure.

http://dx.doi.org/10.3174/ajnr.A3312 
From a genetic and phenotypic point of view, patients with $\mathrm{PNH}$ are also a heterogeneous group. The classic X-linked form is usually caused by mutations of the FLNA gene. ${ }^{11}$ A rare autosomal recessive form is caused by mutations in the ARFGEF2 gene $^{12}$ and is characterized by microcephaly and delayed myelination in addition to $\mathrm{PNH}$. Indeed, $\mathrm{PNH}$ are found in many syndromes; associations with several single-gene disorders, chromosomal anomalies ${ }^{2}$ and some presumably disruptive causes ${ }^{13,14}$ have been published. This heterogeneity suggests that genetic and nongenetic processes may cause PNH. Some authors have noted that those nodules located in the posterior segments of lateral ventricles seem to be more frequently associated with other developmental brain anomalies. ${ }^{15,16}$ To test our hypothesis that the location or number of $\mathrm{PNH}$ determines associations with specific brain malformations, we undertook this retrospective study of MR images in a large series of patients with $\mathrm{PNH}$.

\section{MATERIALS AND METHODS}

MR images of a group of patients with epilepsy that were reviewed by the senior author during the past 12 years were retrospectively examined. This study was approved by the institutional review boards of the University of California at San Francisco and Boston Children's Hospital; patients or their guardians provided written consent when they joined this study. Review of MR images of 1860 patients yielded 200 with multiple PNH; 89 were males (44.5\%), 91 females $(45.5 \%)$ and the sex of 20 patients (10\%) was not known because their records, having been reviewed early in the course of the study, were no longer available. The average age at the time of imaging was 7.9 years, with a range from fetal age to 70 years. Clinical information on the patients was limited, but most were initially referred for epilepsy, often with developmental delay or mental retardation. Because the images were acquired during $>12$ years in different centers, by using different types of MR imaging scanners and different protocols, the quality was rather variable. The MRI of 189 patients (94.5\%) included, at a minimum, T1- and T2-weighted sequences acquired in sagittal and axial planes. In the remaining 11 patients (5.5\%), only 1 sequence was obtained, in a single plane; most commonly, the sagittal series was not acquired (in 7 patients, 3.5\%). Two patients (1\%) were studied only by fetal MR imaging. All of the examinations were reviewed and reported independently by 3 experienced neuroradiologists (G.G., L.V., and A.J.B.); a consensus was reached when any disagreement was encountered.

\section{Classification of Periventricular Nodular Heterotopia}

The number, side, and location of the nodules of PNH were recorded for each patient. By number, they were classified as $\leq 5$ nodules (group 1), 6-10 nodules (group 2), or $>10$ (group 3). Unilateral PNH (uPNH) versus bilateral PNH (bPNH) was recorded. PNH location was classified by the ventricular segments affected (Fig 1). Those in the frontal horns and/or bodies of the lateral ventricles were classified as aPNH, while $\mathrm{pPNH}$ referred to heterotopia located only in the trigones, temporal horns, or occipital horns of the lateral ventricles. Heterotopia located in all of the ventricular segments was classified as $\mathrm{dPNH}$. If heterotopia were few (group 1) and scattered, they were classified as isolated and not included in further analyses. We did not separate a FLNA- like pattern (PNH in the frontal horns, bodies, trigones, and occipital horns but not in the temporal horns) as separate from other patterns because genetic analyses were not available in most of our patients and the associated findings of megacisterna magna and mild vermian hypoplasia are well-described. ${ }^{17,18}$

\section{Classification of Associated Disorders}

Malformations of cortical development associated with the presence of PNH were identified and recorded according to the recent classification by Barkovich et $\mathrm{al}^{1}{ }^{1}$ as disorders of proliferation, migration, or postmigrational organization. Acquired postnatal injury was also recorded.

The volume of white matter and degree of myelination were subjectively assessed. The authors reviewed MR imaging scans of 20 consecutive patients ranging in age from 8 days to 21 years, imaged for seizures, cervical lymphadenopathy, abnormal vision, headaches, developmental delay, or Chiari I malformation (1 patient) to measure the thickness of the band of WM between the most posteromedial margin of both Sylvian fissures and the lateral border of the adjacent ventricular trigone on axial images. The cortex and WM appeared morphologically normal in all. The mean distance was $9.6 \mathrm{~mm}$, and all had measurements of $\geq 8.3 \mathrm{~mm}$. The WM volume was considered to have decreased if the thickness of the WM was $<7$ $\mathrm{mm}$ between the most posteromedial aspect of the Sylvian fissure and the adjacent ventricular margin in the T1 axial sequence (Fig $2 F$ ). In case of $\mathrm{PNH}$ along the trigonal margin, the thickness of WM band was measured up to the lateral margin of the heterotopic nodules. Myelination was compared with the milestones established by Barkovich et al, ${ }^{19}$ depending on the age of the patient. Each of the 3 telencephalic commissures (CC, anterior commissure, and hippocampal commissure) was assessed as per Hetts et al. ${ }^{20}$ The CC was classified as normal, absent, hypogenetic, or hypoplastic. The anterior commissure was classified visually as normal, absent, small (hypoplastic), or thick. The hippocampal commissure was classified as present or absent.

The basal ganglia and thalami were classified as normal, absent, dysmorphic, atrophic, damaged, or containing T2/FLAIR hyperintensities. Because most imaging studies did not allow detailed analyses of the hippocampi, the hippocampi were classified as normal or (if alterations of size or morphology were present) abnormal. The pituitary gland was judged abnormal if the anterior lobe was absent or hypoplastic (anterior lobe of the same size or smaller than posterior lobe) or the posterior lobe was ectopic or absent. The olfactory apparatus was classified as abnormal if the olfactory sulci were shallow or if the olfactory bulbs or nerves were hypoplastic. The mid/hindbrain (brain stem, cerebellar hemispheres, and vermis) findings were recorded as normal or (if absent, hypoplastic, dysmorphic, or atrophic) abnormal. T2/FLAIR hyperintensity was considered abnormal. The presence or absence of megacisterna magna was also recorded.

\section{Statistical Analysis}

For statistical comparisons in $2 \times 2$ contingency tables, the Fisher exact test was used, and for those greater than $2 \times 2$, the $\chi^{2}$ test was used. A value of $P<.05$ was considered significant. 

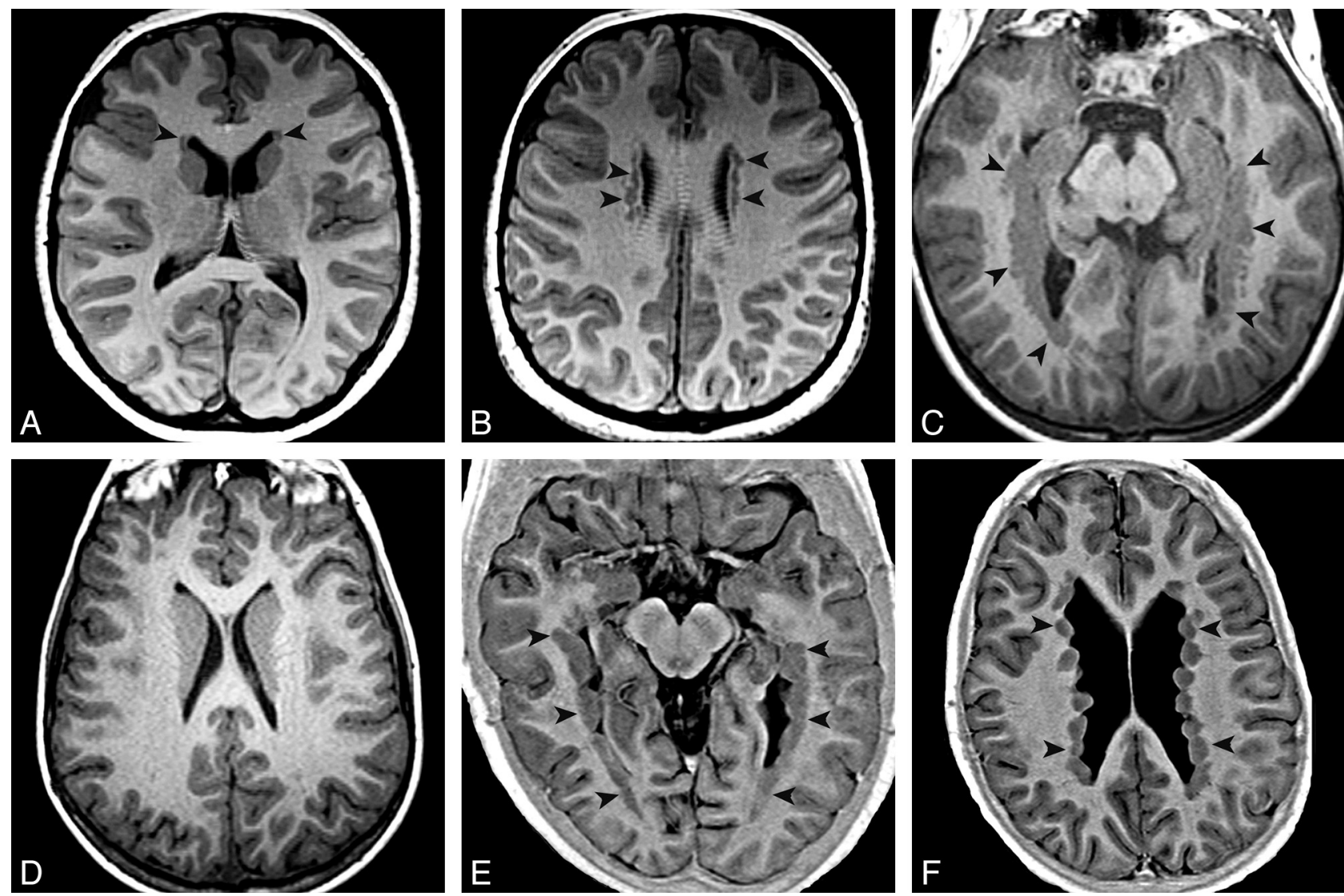

FIG 1. PNH main patterns evaluated with $T 1$ inversion recovery-weighted image. Arrowheads show locations of heterotopia. Axial images show an aPNH pattern with nodules restricted to the frontal horns $(A)$ and bodies $(B)$. Axial images demonstrate a pPNH pattern. Nodules are lining trigones and temporal and occipital horns bilaterally $(C)$, sparing the frontal horns and bodies $(D)$. $E$ and $F$, Axial images show a dPNH pattern with nodules lining all of the walls of the lateral ventricles.

\section{RESULTS}

uPNH were identified in 42 patients $(21 \%)$ and bPNH in 158 (79\%). By number, 63 studies $(31.5 \%)$ were group $1(<5 \mathrm{PNH})$, $39(19.5 \%)$ were group $2(5-10 \mathrm{PNH})$, and 98 (49\%) were group $3(>10 \mathrm{PNH})$. aPNHs were found in 43 patients $(21.5 \%)$; pPNHs, in 58 (29\%); and $\mathrm{dPNH}$, in $32(16 \%)$. The remaining patients, with a few scattered PNHs, were not further classified. The location of the nodules in the ventricular segments is found in On-line Table 1, and the distribution of isolated PNH, in On-line Table 2.

Cortical malformations were present in 66 patients $(33 \%$, Online Table 3). Disorders secondary to abnormal neuronal migration (Barkovich group $\mathrm{II}^{1}$ ) were most frequent (37 cases, 18.5\%), including Walker-Warburg syndrome (2 patients), lissencephalypachygyria (4 patients), subcortical heterotopia (17 patients), and subcortical heterotopia with polymicrogyria or schizencephaly (14 patients). Disorders secondary to abnormal neuronal proliferation (Barkovich group $\mathrm{I}^{1}$ ) were next in frequency, with 16 cases (8\%). Microcephaly with a simplified gyral pattern was diagnosed in 11 cases; 2 patients had microcephaly and a normal sulcation pattern, while 2 had microcephaly with polymicrogyria. One patient had cortical dysplasia in the temporal lobe. Disorders due to abnormal postmigrational cortical organization (Barkovich group III) were observed in 13 patients (6.5\%), all polymicrogyria (including 1 with schizencephaly). Malformations of the cerebral cortex were significantly more frequent in uPNH (52.4\%) than in
bPNH, $(27.2 \%, P=.003)$; cortical malformations were significantly more frequent in pPNH (51.7\%) compared with aPNH, $(14.0 \%, P=.0001)$ and in pPNH compared with $\mathrm{dPNH}(28.1 \%$, $P=.045$; Fig 2). No significant differences were found between the type of cortical malformation and the location pattern of the nodules $(P=.131)$.

Reduced volume of cerebral WM was noticed in 138 patients (69\%). A significant difference in the incidence of reduced WM volume was found between aPNH (51.2\%) and pPNH (75.9\%, $P=.012)$ and between aPNH and dPNH (84.4\%, $P=.003)$. Delayed myelination was seen in $11.5 \%$ of cases with PNH. No significant differences were found among the groups with different patterns of PNH (On-line Table 4).

On-line Table 5 shows the frequency of abnormalities in forebrain commissures in relation to patterns of PNH. Significant differences were found only when comparing the frequency of abnormalities of the CC in aPNH (51.2\%) with those in $\mathrm{dPNH}$ (80.6\%, $P=.014$; Fig 3$)$. Although the frequency of agenesis and hypogenesis was similar in both $\mathrm{PNH}$ patterns, the greater difference arose when comparing the percentages of hypoplasia: $43.8 \%$ in $\mathrm{dPNH}$ and $23.3 \%$ in aPNH. Agenesis of the 3 main forebrain commissures was found in 2 patients (1\%), each with $>10$ PNHs located bilaterally in the bodies, trigones, and temporal horns (On-line Fig 1).

Abnormalities were common in the brain stem (19.5\%), 

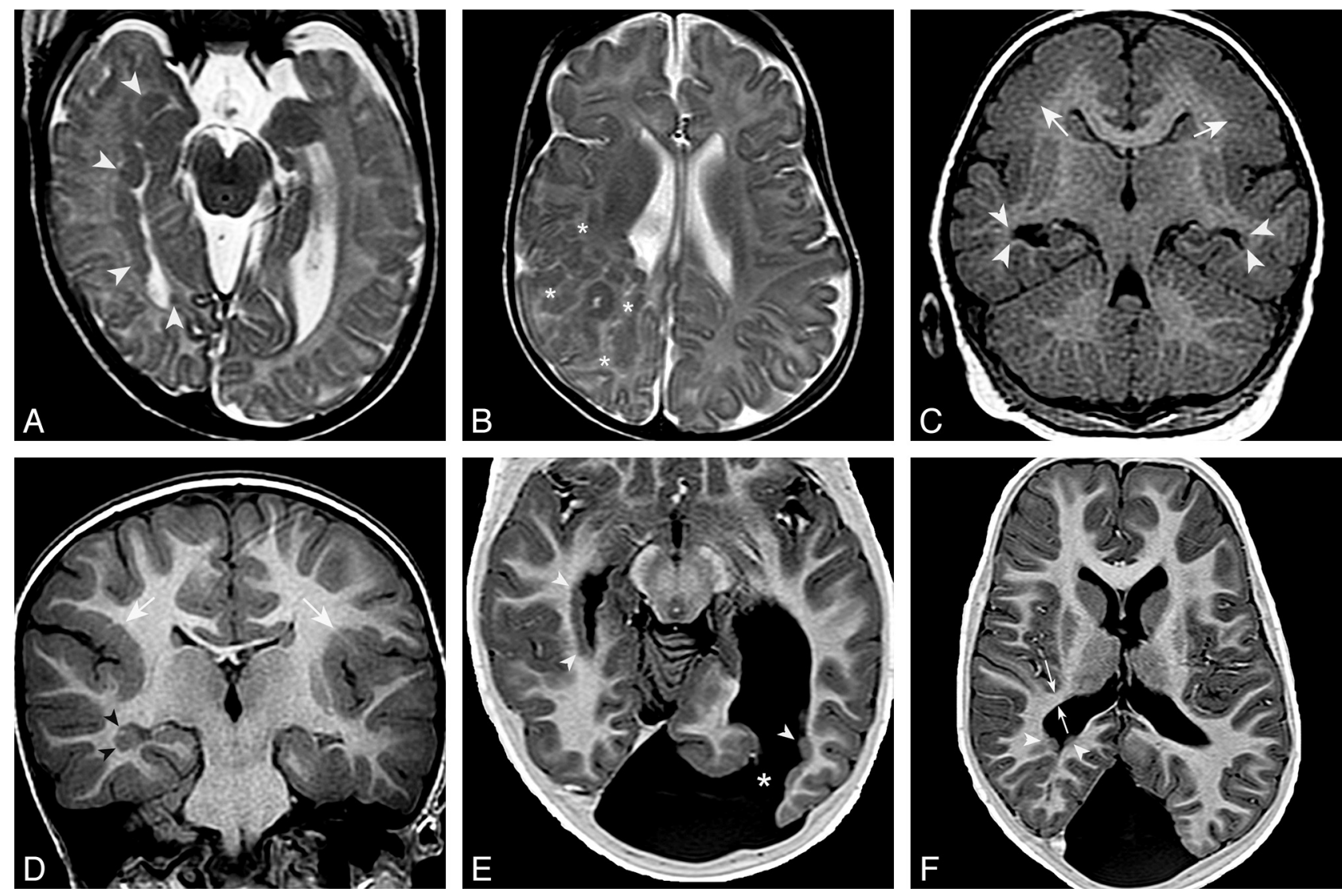

FIG 2. pPNH and malformations of cortical development. Axial T2 FSE images show PNH lining the wall of the right temporal and occipital horns and trigone (arrowheads in A) and a large region of subcortical heterotopia in the right frontoparietal region (asterisks in B). C, Coronal T1 inversion recovery-weighted image demonstrates bilateral PNH in the temporal horns (arrowheads) and bilateral parietal pachygyria (arrows). $D$, Coronal inversion recovery-weighted image shows right temporal $\mathrm{PNH}$ (arrowheads) and bilateral perisylvian polymicrogyria (white arrows). $E$ and $F$, Axial $\mathrm{Tl}$ inversion recovery-weighted images demonstrate bilateral pPNH (arrowheads) and a left occipital schizencephaly (asterisk in E). White arrows in $F$ show the thickness of the WM band measured between the most posteromedial margin of Sylvian fissure and the lateral border of the ventricular trigone. In this case, it was diminished $(5 \mathrm{~mm})$.
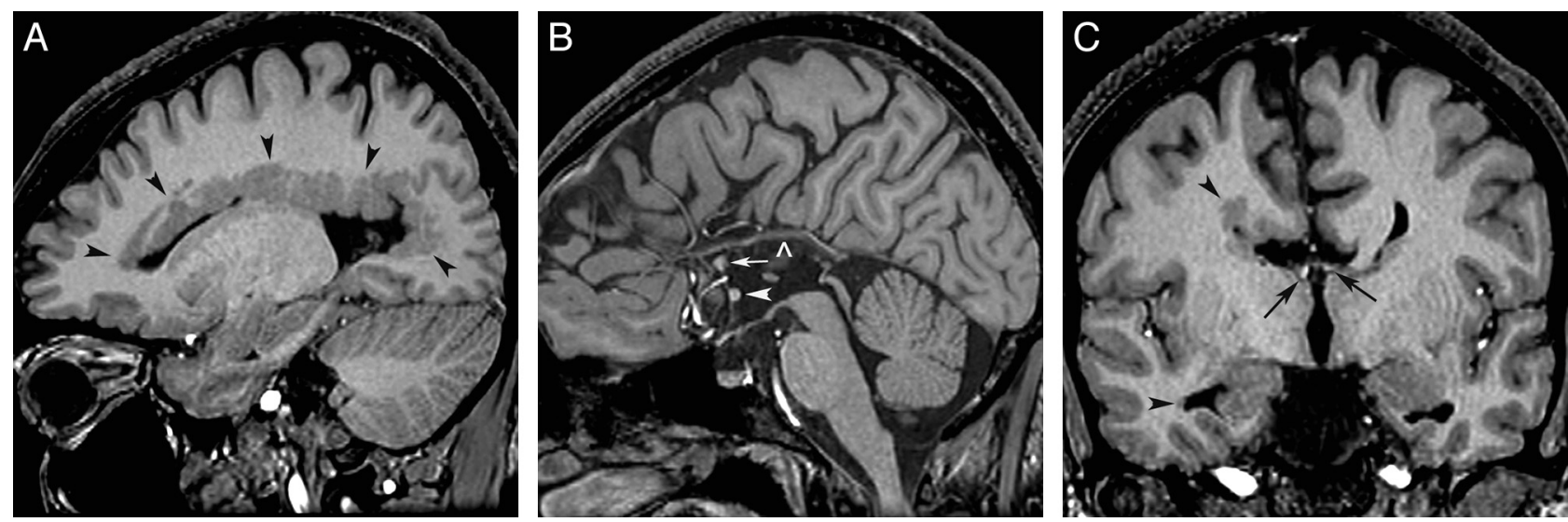

FIG 3. Heterotopia and commissure anomalies. $\mathrm{T}$ inversion recovery-weighted images in a 12-year-old boy with dPNH on the right cerebral hemisphere. A, Right parasagittal image shows multiple PNH lining the entire margin of the lateral ventricle. $B$, Sagittal image demonstrates agenesis of the corpus callosum with a thick anterior commissure (arrowhead) and an anteriorly positioned hippocampal commissure (arrow). A vascular structure is running along of the top of the third ventricle (open arrowhead). C, Coronal image shows moderate white matter volume reduction in the right hemisphere. The fornices are properly located at the roof of the third ventricle (arrows). PNH is seen in the margin of the frontal and temporal horns (arrowheads).

cerebellar vermis (28\%), and cerebellar hemispheres (15\%; Online Table 6). Malformations of the brain stem (31.0\% versus $9.3 \%, P<.014)$ and vermis $(37.9 \%$ versus $14.0 \%, P=.007)$ were more strongly associated with pPNH than aPNH, respectively
(Fig 4). Cerebellar hemispheric malformations were associated more strongly with many PNHs (group 3, 21.4\%) than fewer (groups 1 and 2, 16.2\%, $P<.017$ ) and with pPNH $(24.1 \%)$ rather than with aPNH $(7.0 \%, P<.031)$. The overall frequency of mega- 

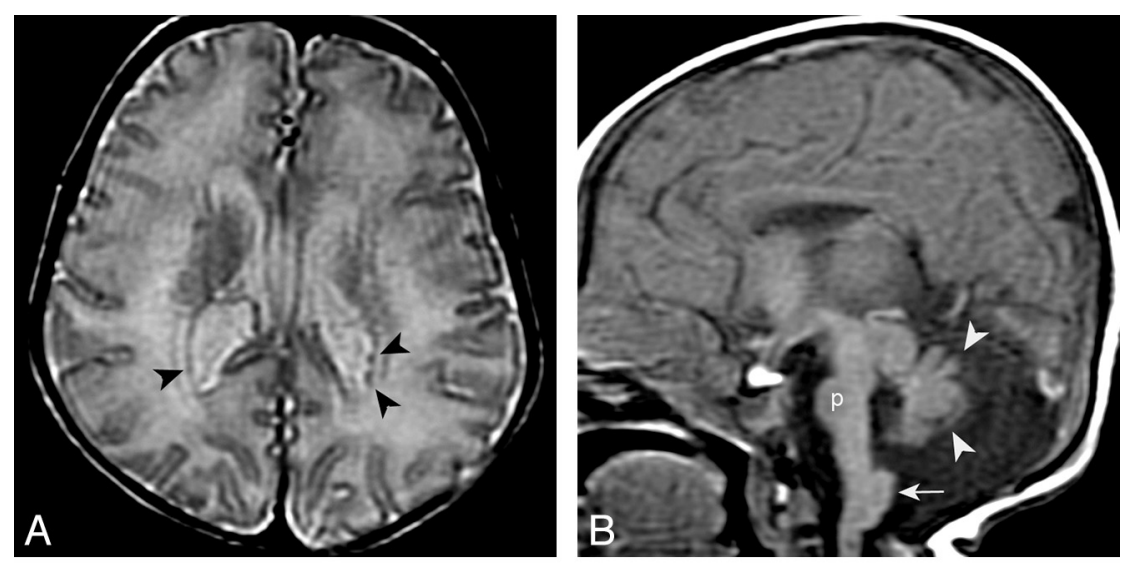

clusively in women and is characterized by bilateral symmetric heterotopic nodules along the frontal horns, bodies, and trigones of the lateral ventricles; the temporal horns are relatively spared and megacisterna magna is common, often in conjunction with cerebellar hypoplasia. ${ }^{17}$ The clinical spectrum of FLNA mutations is wide, however, and missense mutations or mosaicism may result in unilateral forms (also sparing the temporal horn) or nonlethal expression in males. ${ }^{16,18}$

$\mathrm{PNH}$ are quite common, and many forms are not associated with FLNA. Of
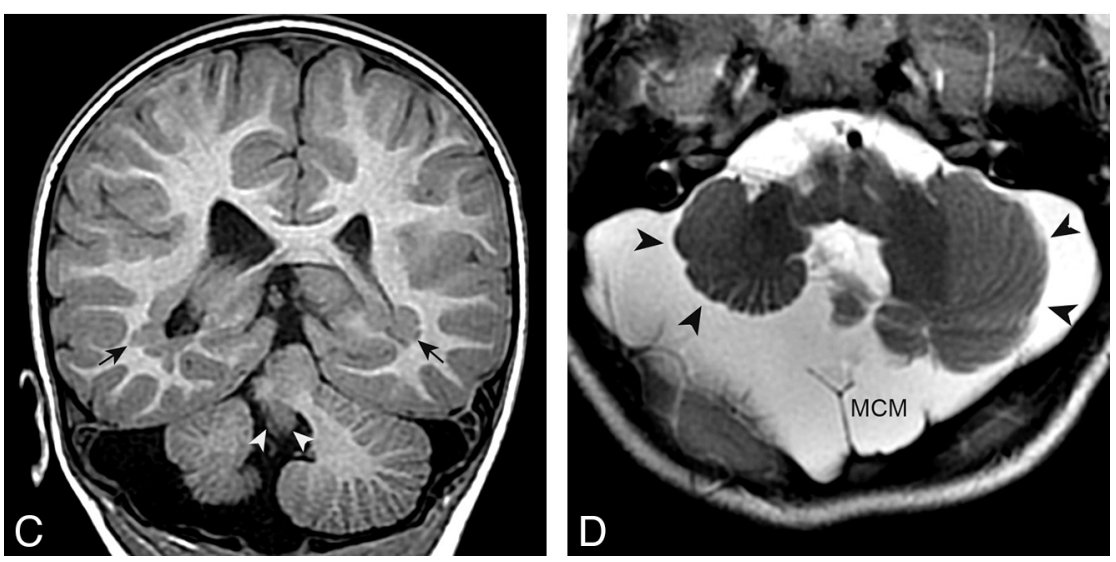

FIG 4. pPNH and posterior fossa anomalies. $A$ and $B$, An 11-day-old boy with microcephaly. Small $\mathrm{PNH}$ are present in the trigones (black arrowheads in $A$ [axial T2 spin-echo-weighted image]). Other findings include a small pons ( $p$ in $B$ [sagittal $T 1$ spin-echo-weighted image]), a hypoplastic and dysmorphic vermis (white arrowheads), and a large inferior cerebellar peduncle (arrow). $C$ and $D, A$ 2-year-old boy with $\mathrm{PNH}$ in the trigones (black arrows in $C$ [coronal $\mathrm{T}$ inversion recovery-weighted image]) and a very small vermis (white arrowheads), dysplastic and small cerebellar hemispheres (black arrowheads), and megacisterna magna (MCM).

182 patients, Parrini et $\mathrm{al}^{16}$ found that only approximately half (54\%) had the classic pattern associated with FLNA mutations. Ten patients ( 7 females, 3 males) had pPNH; all had severe cerebellar vermis hypoplasia, 8 had moderate-to-severe cerebellar hemisphere hypoplasia, and 6 had callosal anomalies. They also reported PNH with polymicrogyria, microcephaly, hydrocephalus, limb anomalies, Ehlers-Danlos syndrome, and fragile $\mathrm{X}$ syndrome. However, they did not perform the detailed analysis of associated brain anomalies that are reported here. They noted neither the brain stem anomalies nor the reduced WM volume identified in this study. This study suggests that patients with PNH can and should be divided into groups based on the locations of heterotopia within the ventricular sys-

cisterna magna in patients with PNH was 12\% (On-Line Table 6); megacisterna magna was more strongly associated with $\mathrm{dPNH}$ (31.3\%) than with aPNH $(0.0 \%, P<.0001)$ or $\mathrm{PPNH}(5.2 \%, P=$ $.0013)$ and with $>10 \mathrm{PNH}(21.4 \%)$ than with $<10 \mathrm{PNH}(6.7 \%$, $P<.0001)$.

Although abnormalities of the basal ganglia, thalami, hippocampi, pituitary/hypothalamus, and myelination were identified among the different patterns of PNH (On-line Tables 4 and 7), no significant differences were associated with $\mathrm{PNH}$ number or location.

\section{DISCUSSION}

The most important finding in this study is that PNH occurs in different patterns that tend to be associated with different types of malformations. Among these patterns, pPNH is particularly distinct because of its significant association with malformations of the cerebral cortex and anomalies of the mid-/hindbrain. The importance of these associations is 2-fold: First, they alert physicians rendering care to these patients to look for specific associated anomalies; and second, they may help in the search for causation, genetic or disruptive.

Many phenotypes of PNH have been described. The classic $\mathrm{X}$-linked form, secondary to FLNA mutations, is seen almost ex- tem. Patients with pPNH demonstrated a significant association with developmental disorders of the cerebral cortex, and disorder of the mid-/hindbrain; the latter may be related to the fact that the occipital and temporal lobes are the posterior portions of the cerebral hemispheres and, therefore, may have more overlap in gene expression with mid-/hindbrain structures than do anterior portions of the cerebrum. Patients with dPNH did not show significant associations with cortical or brain stem anomalies but did have significant association with mid-/hindbrain anomalies. The low frequency of megacisterna magna in patients with pPNH also supports it as a different malformation syndrome, distinguishing it from aPNH and $\mathrm{dPNH}$, and the form associated with FLNA mutations.

Patients with pPNH pattern had significant reduction in WM volume. It is known that some migration disorders, such as lissencephalies, have additional defects in axonal connectivity, ${ }^{21}$ and it has been postulated that causative genes are implicated in both neuronal migration and axon outgrowth. ${ }^{22}$ We may hypothesize, by extension, that this may also be valid for heterotopia and that those phenotypes that have greater association with cortical (neuronal) disorders (eg, pPNH) will show a greater frequency of connectivity abnormalities and, thus, diminished WM.

The reasons for the differences among the patterns of $\mathrm{PNH}$ are AJNR Am J Neuroradiol 34:877-83 Apr 2013 www.ajnr.org 881 
not known. Although it was initially thought to be the result of abnormal neuronal migration, recent studies have suggested that PNH might, instead, be the result of damage to the neuroependyma, which causes disruption of the attachment of radial glia; neurons in the regions of such disruption would not be able to migrate into the intermediate zone and cortex but, rather, would remain as nodules of neurons close to the ventricular surface. ${ }^{5}$ Mechanical neuroependymal disruptions, in addition to genetic causes, might result in PNH if it occurs before the cessation of neuronal proliferation and the beginning of migration. The occurrence of PNH from disruptions might help to explain the association of polymicrogyria and schizencephaly (both believed to be disruptive in many cases ${ }^{1}$ ) with $\mathrm{PNH}$. One might speculate that the posterior location might be related to the disproportionate ventricular dilation often seen in the temporal horns and trigones of children with hydrocephalus or to mutation of genes that are disproportionately expressed within the temporal and occipital ventricular zones. This hypothesis has the potential to be an interesting area of research for the future. As mentioned briefly above, the association of pPNH with mid-/hindbrain malformations might also suggest that genes expressed more strongly in the posterior telencephalon (and therefore more likely to be expressed in the mid-/hindbrain) might be likely candidates in this subgroup of patients with $\mathrm{PNH}$.

dPNH has a similar phenotype to that in patients with FLNA mutations, being significantly associated with megacisterna magna and callosal abnormalities. Indeed, it is likely that some patients with dPNH have mutations in the FLNA gene. Currently, most dPNH syndromes are clinically defined ${ }^{1}$; at this time, only 2 chromosomal anomalies have been related to this phenotype: duplication 5p15.33 and deletion 6q27. The involved genes and their functions are not understood nor is the cause for the PNH in affected patients. No genes associated with aPNH have yet been described.

One limitation of this study is the lack of comprehensive clinical histories and genetic analyses in the patients. However, this does not weaken the fundamental finding that specific distributions of PNH have strong associations with specific malformation complexes. Another potential weakness is the retrospective nature of this review: wide variability in MR imaging protocols, lack of sequences in all planes, and disparate quality in images did not allow a comprehensive evaluation of brain structures in every patient. (An ideal MR imaging protocol for evaluating brain malformations should include a volumetric sagittal T1 inversion recovery-weighted image with reformat in axial and coronal planes, an axial T2 spin-echo-weighted image, a volumetric coronal T2 FSE-weighted image with reformat in the sagittal plane, and DTI for study of WM tracts. In patients older than 1 year, a T2-FLAIR sequence can be added.) Nonetheless, the large number of subjects in this cohort provides solid and valid results, allowing good morphologic characterization of the $\mathrm{PNH}$ and the accompanying disorders. These results and their implications will, hopefully, encourage further research in this field.

\section{CONCLUSIONS}

The results of this study suggest that $\mathrm{PNH}$ should not be considered a single uniform entity. Indeed, it may be useful to consider
aPNH, pPNH, and dPNH as distinct phenotypes within the spectrum of patients with $\mathrm{PNH}$. Specific PNH patterns with their associated malformations may be considered as specific malformation complexes, likely with specific genetic causes in addition to the known X-linked and autosomal recessive disorders. Nongenetic disruptive causes might be considered in some cases, especially in $\mathrm{pPNH}$, which is associated with some known disruptive developmental disorders. ${ }^{13,14}$

\section{ACKNOWLEDGMENTS}

We thank the clinicians, researchers, and families who have volunteered their time and effort to provide imaging and to participate in the research to make this article possible.

Disclosures: Annapurna Poduri—RELATED: Grant: National Institute of Neurological Disorders and Stroke (NINDS), ${ }^{*}$ UNRELATED: Grants/Grants Pending: NINDS.* Christopher Walsh—RELATED: Grant: NINDS, * Howard Hughes Medical Institute, ${ }^{*}$ Comments: This is our grant funding, which I presume is not regarded as any sort of conflict of interest, UNRELATED: Board Membership: Generation Health, Comments: I think that this has no relationship whatsoever to the work, but I disclose it for completeness. I am a member of the clinical advisory board for this company, whose interests are in genetic testing, but which has had no interest in the subject of this article, Consultancy: Roche, Novartis, Comments: I have spoken at or done minor consulting for each of these entities. I can think of no conceivable relationship between their financial interests and the work discussed in this article but merely disclose them for completeness, Payment for Lectures (including service on Speakers Bureaus): Pfizer, Comments: I have spoken at a symposium at Pfizer. I can think of no conceivable relationship between their financial interests and the work discussed in this article but merely disclose them for completeness. Anthony James Barkovich-RELATED: Grant: National Institutes of Health,* Comments: grant: Human Epilepsy Genetics: Neuronal Migration Disorders (R01 NS 035129). *Money paid to the institution.

\section{REFERENCES}

1. Barkovich AJ, Guerrini R, Kuzniecky RI, et al. A developmental and genetic classification for malformations of cortical development: update 2012. Brain 2012;135(pt 5):1348-69

2. Guerrini R, Parrini E. Neuronal migration disorders. Neurobiol Dis 2010;38:154-66

3. Garbelli R, Rossini L, Moroni RF, et al. Layer-specific genes reveal a rudimentary laminar pattern in human nodular heterotopia. $\mathrm{Neu}$ rology 2009;73:746-53

4. Sarkisian MR, Bartley CM, Rakic P. Trouble making the first move: interpreting arrested neuronal migration in the cerebral cortex. Trends Neurosci 2008;31:54-61

5. Ferland RJ, Batiz LF, Neal J, et al. Disruption of neural progenitors along the ventricular and subventricular zones in periventricular heterotopia. Hum Mol Genet 2009;18:497-516

6. Lu J, Tiao G, Folkerth R, et al. Overlapping expression of arfgef 2 and filamin a in the neuroependymal lining of the lateral ventricles: insights into the cause of periventricular heterotopia. J Comp Neurol 2006;494:476-84

7. Battaglia G, Chiapparini L, Franceschetti S, et al. Periventricular nodular heterotopia: classification, epileptic history, and genesis of epileptic discharges. Epilepsia 2006;47:86-97

8. Battaglia G, Franceschetti S, Chiapparini L, et al. Electroencephalographic recordings of focal seizures in patients affected by periventricular nodular heterotopia: role of the heterotopic nodules in the genesis of epileptic discharges. J Child Neurol 2005;20:369-77

9. de Wit MC, Schippers HM, de Coo IF, et al. Absence epilepsy and periventricular nodular heterotopia. Seizure 2010;19:450-52

10. Leventer RJ, Guerrini R, Dobyns WB. Malformations of cortical development and epilepsy. Dialogues Clin Neurosci 2008;10:47-62

11. Fox JW, Lamperti ED, Eksioglu YZ, et al. Mutations in filamin 1 prevent migration of cerebral cortical neurons in human periventricular heterotopia. Neuron 1998;21:1315-25 
12. Sheen VL, Ganesh VS, Topcu M, et al. Mutations in arfgef2 implicate vesicle trafficking in neural progenitor proliferation and migration in the human cerebral cortex. Nat Genet 2004;36:69-76

13. Barth PG, van der Harten JJ. Parabiotic twin syndrome with topical isocortical disruption and gastroschisis. Acta Neuropathol 1985;67:345-49

14. Okumura A, Lee T, Shimojima K, et al. Brainstem disconnection associated with nodular heterotopia and proatlantal arteries. $\mathrm{Am} \mathrm{J}$ Med Genet A 2009;149A:2479-83

15. Barkovich AJ, Raybaud CA. Congenital malformations of the brain and skull. In Barkovich AJ, Raybaud CA, eds. Pediatric Neuroimaging. Philadelphia: Wolters Kluwer Health/Lippincott Williams \& Wilkins; 2011:367-568

16. Parrini E, Ramazzotti A, Dobyns WB, et al. Periventricular heterotopia: phenotypic heterogeneity and correlation with filamin a mutations. Brain 2006;129:1892-906

17. Poussaint TY, Fox JW, Dobyns WB, et al. Periventricular nodular heterotopia in patients with filamin-1 gene mutations: neuroimaging findings. Pediatr Radiol 2000;30:748-55

18. Guerrini R, Mei D, Sisodiya S, et al. Germline and mosaic mutations of fln1 in men with periventricular heterotopia. Neurology 2004;63:51-56

19. Barkovich AJ, Mukherjee, P. Normal development of the neonatal and infant brain, skull and spine. In: Barkovich AJ, Raybaud CA, eds. Pediatric Neuroimaging. Philadelphia: Wolters Kluwer Health/Lippincott Williams \& Wilkins; 2011:20-80

20. Hetts SW, Sherr EH, Chao S, et al. Anomalies of the corpus callosum: an MR analysis of the phenotypic spectrum of associated malformations. AJR Am J Roentgenol 2006;187:1343-48

21. Kara S, Jissendi-Tchofo P, Barkovich AJ. Developmental differences of the major forebrain commissures in lissencephalies. AJNR Am J Neuroradiol 2010;31:1602-07

22. Liu JS. Molecular genetics of neuronal migration disorders. Curr Neurol Neurosci Rep 2011;11:171-78 